\title{
APPLICATION OF THE CONTROL VOLUME METHOD USING THE VORONOI POLYGONS FOR NUMERICAL MODELING OF BIO-HEAT TRANSFER PROCESSES
}

\author{
Mariusz Ciesielski \\ Czestochowa University of Technology, Częstochowa, Poland; e-mail: mariusz.ciesielski@icis.pcz.pl \\ BOHDAN MOCHNACKI \\ Higher School of Labour Safety Management, Katowice, Poland; e-mail: bmochnacki@wszop.edu.pl
}

\begin{abstract}
In the paper, the problem concerning the numerical modeling of thermal processes in the domain of a biological tissue being in thermal contact with the environment is discussed. The changing ambient temperature causes that the non-steady heat transfer process is considered. The cross-section of the forearm (2D problem) is treated as a non-homogeneous domain in which the sub-domains of skin tissue, fat, muscle and bone are distinguished. From the mathematical point of view, the boundary-initial problem described by the system of energy equations (the Pennes equations), boundary conditions on the external surface of the system, boundary conditions on the surfaces limiting the successive sub-domains and the initial condition is analyzed. At the stage of numerical computations, the Control Volume Method using the Voronoi polygons is applied. In the final part of the paper, examples of computations are shown.
\end{abstract}

Keywords: bioheat transfer, numerical modeling, Control Volume Method, Voronoi's diagram

\section{Introduction}

The thermal processes proceeding in the domain of a biological tissue are, as a rule, described by the well known Pennes equations (e.g. Mochnacki and Majchrzak, 2003; Majchrzak, 2013). The typical heat diffusion equation is supplemented by components determining the capacities of internal heat sources connected with the blood perfusion and metabolism. The mathematical form of perfusion heat source results from the assumption that the tissue is supplied by a large number of blood capillaries uniformly distributed in the area under consideration. In the case of tissue freezing modeling, the right hand side of the Pennes equation should be supplemented by the third internal heat source controlling the evolution of latent heat (e.g. Majchrzak et al., 2011). The Pennes equation constitutes a basis of the so-called tissue models (Mochnacki and Majchrzak, 2003; Majchrzak, 2013; Majchrzak et al., 2011; Mochnacki and Piasecka-Belkhayat, 2013). Atkin et al. (1994) talk over different bio-heat transfer tissue models and conclude that the Pennes equation is the best approach to the modeling of bio-heat transfer because of its simplicity.

In literature, one can also find the so-called vascular models (Majchrzak, 2013; Zhu and Weinbaum, 1995; Wang et al., 2007). The vascular models are applied because of the need to include the thermally significant blood vessels of considerable size. In this paper, the presence of blood vessels (arteries and veins) in the domain considered is taken into account, but the blood temperature is optionally established on the basis of literature data (it is not calculated), and the model presented belongs rather to the tissue ones.

There have recently been attempts to use descriptions of thermal processes in the biological tissue on the basis of porous media theory. The domain considered is divided into two parts, that this the vascular and extravascular region (cells and interstitial tissue). The blood vessels 
are treated as voids, while the remaining area as the matrix (solid body). The equations concerning the vascular and extravascular regions contain the parameter $\varepsilon$ (porosity) (Zhang, 2009; Nakayama and Kuwahara, 2008; Khaled and Vafai, 2003). Assuming certain simplifications, the above mentioned equations can be substituted by a single equation close to the dual phase lag model (Khaled and Vafai, 2003).

In this paper, the cross section of the forearm (middle part) shown in Fig. 1 (Schuenke et al., 2010) is considered. The domain is non-homogeneous and constitutes a composition of skin, fat, muscle, bone and blood vessels.
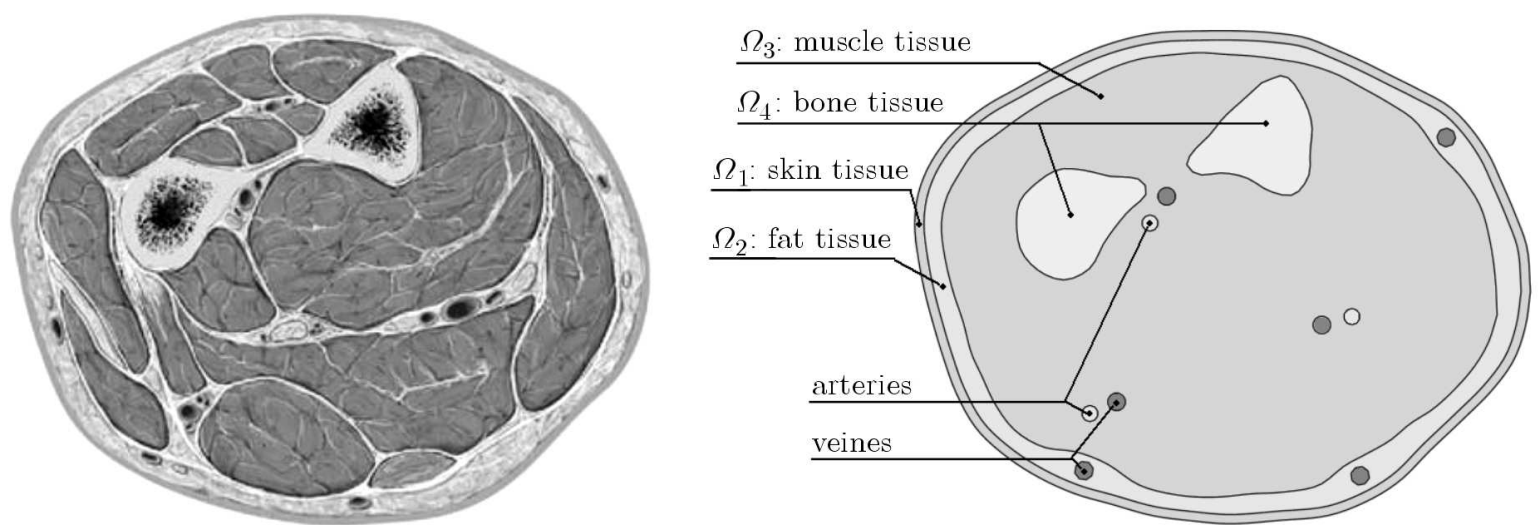

Fig. 1. Forearm cross section and a simplified 2D geometrical model

The mathematical model of thermal processes proceeding in the area considered subjected to the time-dependent external heat source is presented in Section 2.

At the stage of numerical modeling, the Control Volume Method using the Voronoi tessellation is used (Domanski et al., 2010). The Voronoi polygons are characterized by the geometric properties of a well meeting the requirements for the shape of control volumes. The details of the approach proposed are discussed in Section 3. In the final part of the paper, examples of computations are presented.

\section{Governing equations}

Heat transfer processes proceeding in the tissue domain are described by the system of the Pennes equations of the following form

$$
c_{e}(T) \frac{\partial T_{e}(x, t)}{\partial t}=\nabla\left[\lambda_{e}(T) \nabla T_{e}(x, t)\right]+Q_{p e r e}(T)+Q_{m e t e}(T) \quad e=1, \ldots, 4
$$

where $e=1, \ldots, 4$ identifies the tissue sub-domains (skin, fat, muscle and bone respectively), $c_{e}$ is the volumetric specific heat, $\lambda_{e}$ is the thermal conductivity, $Q_{p e r}$ and $Q_{m e t}$ are the capacities of volumetric internal heat sources connected with the blood perfusion and metabolism, $\left[\mathrm{W} / \mathrm{m}^{3}\right], T, x=\left\{x_{1}, x_{2}\right\}, t$ denotes temperature, spatial co-ordinates and time, respectively. The perfusion heat source is given by the formula

$$
Q_{\text {pere } e}(T)=c_{b} G_{b e}(T)\left[T_{b}-T_{e}(x, t)\right] \quad T_{b}=\frac{1}{2}\left(T_{b \text { artery }}+T_{b v e i n}\right)
$$

where $G_{b e}$ is the blood perfusion $\left[\mathrm{m}^{3}\right.$ blood/( $\mathrm{s} \mathrm{m}^{3}$ tissue $\left.)\right], c_{b}$ is the blood volumetric specific heat and $T_{b \text { artery }}$ and $T_{b \text { vein }}$ are the arterial and vein blood temperatures. The metabolic heat source $Q_{m e t}$ can be treated both as a constant value and a temperature-dependent function. 
On the contact surface between the tissue sub-domains, the IV type of boundary conditions are assumed

$$
x \in \Gamma_{k-l}:\left\{\begin{array}{l}
-\lambda_{k} \frac{\partial T_{k}(x, t)}{\partial n}=-\lambda_{l} \frac{\partial T_{l}(x, t)}{\partial n} \\
T_{k}(x, t)=T_{l}(x, t)
\end{array} \quad(k, l) \in\{(1,2),(2,3),(3,4)\}\right.
$$

where $\partial / \partial n$ denotes the normal derivative.

On the outer surface of the skin $(e=1)$, the III type of boundary condition is taken into account

$$
x \in \Gamma_{\text {out }}: \quad-\lambda_{1} \frac{\partial T_{1}(x, t)}{\partial n}=-\alpha_{\text {out }}\left[T_{\text {amb }}(t)-T_{1}(x, t)\right]
$$

where $\alpha_{\text {out }}$ is the heat transfer coefficient, $T_{a m b}$ is the ambient temperature. The same type of boundary conditions is given on the contact surfaces between the blood vessels and soft tissue sub-domains, in particular

$$
x \in \Gamma_{\text {artery }}: \quad-\lambda_{3} \frac{\partial T_{3}(x, t)}{\partial n}=-\alpha_{\text {artery }}\left[T_{\text {bartery }}-T_{3}(x, t)\right]
$$

and

$$
x \in \Gamma_{\text {vein }}: \quad-\lambda_{e} \frac{\partial T_{e}(x, t)}{\partial n}=-\alpha_{\text {vein }}\left[T_{b v e i n}-T_{e}(x, t)\right] \quad e=\{2,3\}
$$

The initial conditions are also given

$$
t=0: \quad T_{e}(x, t)=T_{\text {steady }}(x) \quad e=1, \ldots, 4
$$

where $T_{\text {steady }}$ is the temperature distribution corresponding to the steady state conditions in the domain considered for the given ambient temperature and the initial external heat transfer coefficient.

\section{Numerical algorithm}

For the purpose of heat transfer modeling in the domain considered, the tissue sub-domains are divided into small cells (control volumes) known as the Voronoi polygons (also called the Thiessen or Dirichlet cells in two dimensions) (Okabe et al., 2000). The polygon that contains the point $x_{i}$ (central point) is denoted by $\Delta V_{i}$, see Fig. 2 . All of the Voronoi regions are convex polygons, and each polygon is defined by lines that bisect the sectors between the central point and its neighbouring points. The bisecting lines and the connection lines are perpendicular to each other (it is very convenient at the stage of CVM equations construction). When we use this rule for every point in the area, the area will be completely covered by adjacent polygons. Many algorithms to construct the Voronoi polygons can be found in literature, see e.g. the Delaunay triangulation (Watson, 1981).

In Fig. 3, an example of the control volume mesh in the domain of the forearm cross-section is shown. The domain is divided into 2966 control volumes. The positions of CV central points close to the contact surface between the tissue sub-domains are analytically determined in order to achieve a better approximation of the tissue shape.

The control volume method (CVM) constitutes an effective tool for numerical computation of heat transfer processes. The domain analyzed is divided into $N$ volumes. The CVM algorithm allows one to find the transient temperature field at the set of nodes corresponding to the 


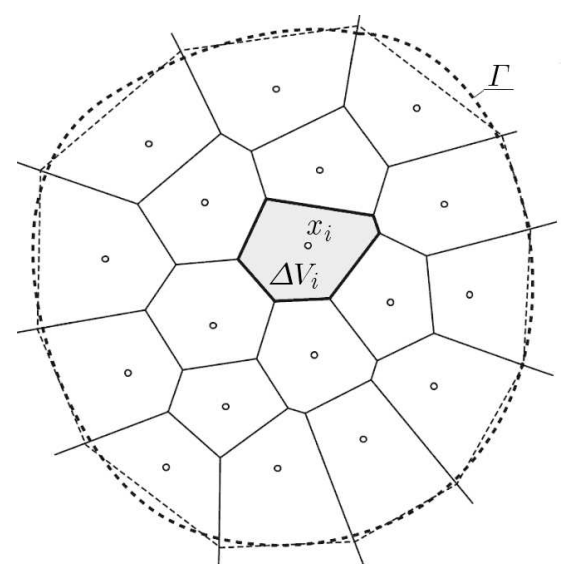

Fig. 2. The Voronoi polygons for a set of arbitrarily distributed points

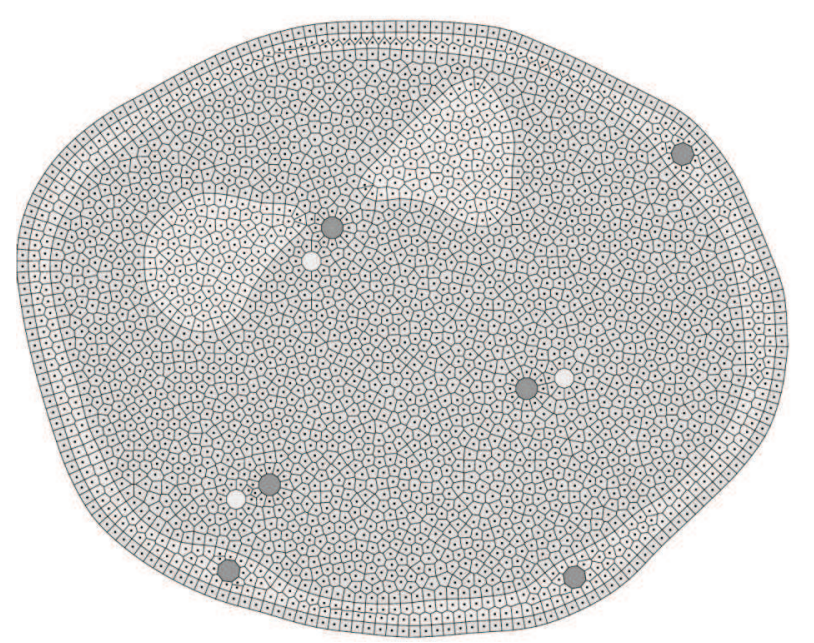

Fig. 3. Control volume mesh: cross-section of the forearm

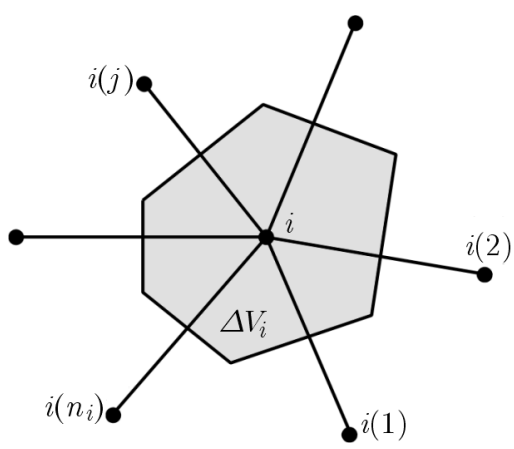

Fig. 4. Control volume $\Delta V_{i}$

central points of the control volumes. The nodal temperatures can be found on the basis of energy balances for the successive volumes.

Let us consider the control volume $\Delta V_{i}$ with the central node $x_{i}$. It is assumed here that the thermal capacities and capacities of the internal heat sources are concentrated at the nodes representing the elements, while the thermal resistances are concentrated on the sectors joining the nodes. The energy balances corresponding to the heat exchange between the analyzed control volume $\Delta V_{i}$ and the adjoining control volumes results from the integration of energy equation (2.1) with respect to time and volume $C V_{i}$. Let us consider the interval of time $\Delta t=t^{f+1}-t^{f}$. 
Then

$$
\begin{aligned}
& \int_{t^{f}}^{t^{f+1}} \int_{C V_{i}} c_{e}(T) \frac{\partial T_{e}(x, t)}{\partial t} d V d t=\int_{t^{f}}^{t^{f+1}} \int_{C V_{i}} \nabla\left[\lambda_{e}(T) \nabla T_{e}(x, t)\right] d V d t \\
& \quad+\int_{t^{f}}^{t^{f+1}} \int_{C V_{i}}\left[Q_{\text {per } e}(T)+Q_{\text {met e }}(T)\right] d V d t
\end{aligned}
$$

Using Gauss-Ostrogradsky's theorem, one obtains

$$
\begin{aligned}
& \int_{t^{f}}^{t^{f+1}} \int_{C V_{i}} c_{e}(T) \frac{\partial T_{e}(x, t)}{\partial t} d V d t=\int_{t^{f}}^{t^{f+1}} \int_{A_{i}} \mathbf{n} \cdot\left[\lambda_{e}(T) \nabla T_{e}(x, t)\right] d A d t \\
& \quad+\int_{t^{f}}^{t^{f+1}} \int_{C V_{i}}\left[Q_{\text {per } e}(T)+Q_{\text {met }}(T)\right] d V d t
\end{aligned}
$$

where $A_{i}$ is the surface (perimeter) limiting the $C V_{i}$. The approximation of the left-hand side of equation (3.2) can be taken in form

$$
\int_{t^{f}}^{t^{f+1}} \int_{C V_{i}} c_{e}(T) \frac{\partial T_{e}(x, t)}{\partial t} d V d t \cong c_{i}^{f}\left(T_{i}^{f+1}-T_{i}^{f}\right) \Delta V_{i}
$$

where $c_{i}^{f}$ is an integral mean of thermal capacity, and this value is approximated by the volumetric specific heat corresponding to the temperature $T_{i}^{f}$. In a similar way, one can approximate the last component in equation (3.2), namely

$$
\int_{t^{f}}^{t^{f+1}} \int_{C V_{i}}\left(Q_{\text {pere }}(T)+Q_{\text {met }}(T)\right) d V d t \cong\left[\left(Q_{p e r}\right)_{i}^{f}+\left(Q_{m e t}\right)_{i}^{f}\right] \Delta V_{i} \Delta t
$$

The term determining the heat conduction between $\Delta V_{i}$ and its neighbourhoods can be written in form

$$
\begin{aligned}
& \int_{t^{f}}^{t^{f+1}} \int_{A_{i}} \mathbf{n} \cdot\left[\lambda_{e}(T) \nabla T_{e}(x, t)\right] d A d t=\int_{t^{f}}^{t^{f+1}}\left(\sum_{j=1}^{n_{i}} \int_{A_{i(j)}} \mathbf{n}_{i(j)} \cdot[\lambda(T) \nabla T(x, t)]_{i(j)} d A_{i(j)}\right) d t \\
& =\int_{t^{f}}^{t^{f+1}}\left(\sum_{j=1}^{n_{i}} \mathbf{n}_{i(j)} \cdot[\lambda(T) \nabla T(x, t)]_{i(j)} A_{i(j)}\right) d t \cong \int_{t^{f}}^{t^{f+1}}\left(\sum_{j=1}^{n_{i}} \lambda_{i j} \frac{T_{i(j)}-T_{i}}{h_{i(j)}} A_{i(j)}\right) d t \\
& =\sum_{j=1}^{n_{i}} \lambda_{i j}^{f} \frac{T_{i(j)}^{f}-T_{i}^{f}}{h_{i(j)}} A_{i(j)} \Delta t
\end{aligned}
$$

where $\lambda_{i j}$ is the mean thermal conductivity between the nodes $i$ and $i(j)$, in particular

$$
\lambda_{i j}=\frac{2 \lambda_{i} \lambda_{i(j)}}{\lambda_{i}+\lambda_{i(j)}}
$$


Such an assumption causes that in the denominators of conductional terms, the well known thermal resistances appear. The energy balance written in convention of 'explicit' scheme takes the form

$$
c_{i}^{f}\left(T_{i}^{f+1}-T_{i}^{f}\right) \Delta V_{i}=\sum_{j=1}^{n_{i}} \lambda_{i j}^{f} \frac{T_{i(j)}^{f}-T_{i}^{f}}{h_{i(j)}} A_{i(j)} \Delta t+\left[\left(Q_{p e r}\right)_{i}^{f}+\left(Q_{m e t}\right)_{i}^{f}\right] \Delta V_{i} \Delta t
$$

from which (introducing $\left(Q_{p e r}\right)_{i}^{f}=\left(G_{b}\right)_{i}^{f} c_{b}\left(T_{b}-T_{i}^{f}\right)$ )

$$
T_{i}^{f+1}=T_{i}^{f}+\frac{\Delta t}{c_{i}^{f} \Delta V_{i}} \sum_{j=1}^{n_{i}} \lambda_{i j}^{f} \frac{T_{i(j)}^{f}-T_{i}^{f}}{h_{i(j)}} A_{i(j)}+\frac{\Delta t}{c_{i}^{f}}\left[\left(G_{b}\right)_{i}^{f} c_{b}\left(T_{b}-T_{i}^{f}\right)+\left(Q_{m e t}\right)_{i}^{f}\right]
$$

or

$$
T_{i}^{f+1}=T_{i}^{f}+\sum_{j=1}^{n_{i}} W_{i(j)}\left(T_{i(j)}^{f}-T_{i}^{f}\right)+\frac{\Delta t}{c_{i}^{f}}\left[\left(G_{b}\right)_{i}^{f} c_{b}\left(T_{b}-T_{i}^{f}\right)+\left(Q_{m e t}\right)_{i}^{f}\right]
$$

where

$$
W_{i(j)}=\frac{\lambda_{i j}^{f} A_{i(j)} \Delta t}{c_{i}^{f} h_{i(j)} \Delta V_{i}}
$$

The stability condition

$$
1-\sum_{j=1}^{n_{i}} W_{i(j)}-\frac{\Delta t\left(G_{b}\right)_{i}^{f} c_{b}}{c_{i}^{f}}>0
$$

(for all $i$ ) allows one to determine the critical time step.

In the case of external $C V$ (the boundary of $C V_{i}$ between the nodes $i$ and $i(j)$ lies on the surface $\Gamma_{\text {out }}, \Gamma_{\text {artery }}$ and $\left.\Gamma_{\text {vein }}\right)$, the following approximation of conductional term in equation (3.5) is used

$$
\mathbf{n}_{i(j)} \cdot[\lambda(T) \nabla T(X, t)]_{i(j)} A_{i(j)} \cong \frac{T_{a}-T_{i}}{\frac{h_{i(j)}}{2 \lambda_{i}}+\frac{1}{\alpha}} A_{i(j)}
$$

where $T_{a}=\left\{T_{a m b}, T_{b \text { artery }}, T_{b \text { vein }}\right\}$ and $\alpha=\left\{\alpha_{\text {out }}, \alpha_{\text {artery }}, \alpha_{\text {vein }}\right\}$, respectively. Then, the coefficient $W_{i(j)}$ in formula (3.10) takes the form

$$
W_{i(j)}=\frac{A_{i(j)} \Delta t}{c_{i}^{f}\left(\frac{h_{i(j)}}{2 \lambda_{i}}+\frac{1}{\alpha}\right) \Delta V_{i}}
$$

and simultaneously $T_{i(j)}^{f}=T_{a}$. One can see that the denominator in formula (3.13) corresponds to the thermal resistance between the central point of $C V_{i}$ and its environment in the $i(j)$ direction.

\section{Example of computations}

The forearm domain stays in the thermal contact with the environment whose temperature is equal to $T_{a m b}=20^{\circ} \mathrm{C}$, while the heat transfer coefficient is $\alpha_{\text {out }}=3.7 \mathrm{~W} /\left(\mathrm{m}^{2} \mathrm{~K}\right)$. The initial temperature distribution is found using the Gauss method (simple iteration method). The thermophysical parameters of the successive sub-domains are taken from Fiala et al. (1999), see Table 1. 
Table 1. Thermal parameters of the tissues

\begin{tabular}{|l|c|c|c|c|c|}
\hline & $\lambda[\mathrm{W} / \mathrm{mK}]$ & $\rho\left[\mathrm{kg} / \mathrm{m}^{3}\right]$ & $c[\mathrm{~J} /(\mathrm{kg} \mathrm{K})]$ & $G_{b}[1 / \mathrm{s}]$ & $Q_{\text {met }}\left[\mathrm{W} / \mathrm{m}^{3}\right]$ \\
\hline \hline Bone & 0.75 & 1357 & 1700 & $0.0000 \cdot 10^{-3}$ & 0 \\
\hline Muscle & 0.42 & 1085 & 3768 & $0.5380 \cdot 10^{-3}$ & 684 \\
\hline Fat & 0.16 & 850 & 2300 & $0.0036 \cdot 10^{-3}$ & 58 \\
\hline Skin & 0.47 & 1085 & 3680 & $1.1000 \cdot 10^{-3}$ & 368 \\
\hline Blood & \multicolumn{7}{|l}{} & & \\
\hline
\end{tabular}

Additionally, the following blood temperatures are assumed: $T_{\text {bartery }}=36^{\circ} \mathrm{C}, T_{\text {bvein }}=35^{\circ} \mathrm{C}$, while $\alpha_{\text {artery }}=\alpha_{\text {vein }}=5000 \mathrm{~W} /\left(\mathrm{m}^{2} \mathrm{~K}\right)$. At the moment $t=0$ the ambient temperature increases to $40^{\circ} \mathrm{C}$ (the heat transfer coefficient is the same). Such a situation may correspond to the input from a room outside in a summer hot day. The numerical simulation concerns the process of tissue heating.

In Fig. 5, the initial temperature distribution is shown. The next figures present temporary solutions for times $15 \mathrm{~min}$. and $300 \mathrm{~min}$. (this result practically corresponds to the steady state conditions).

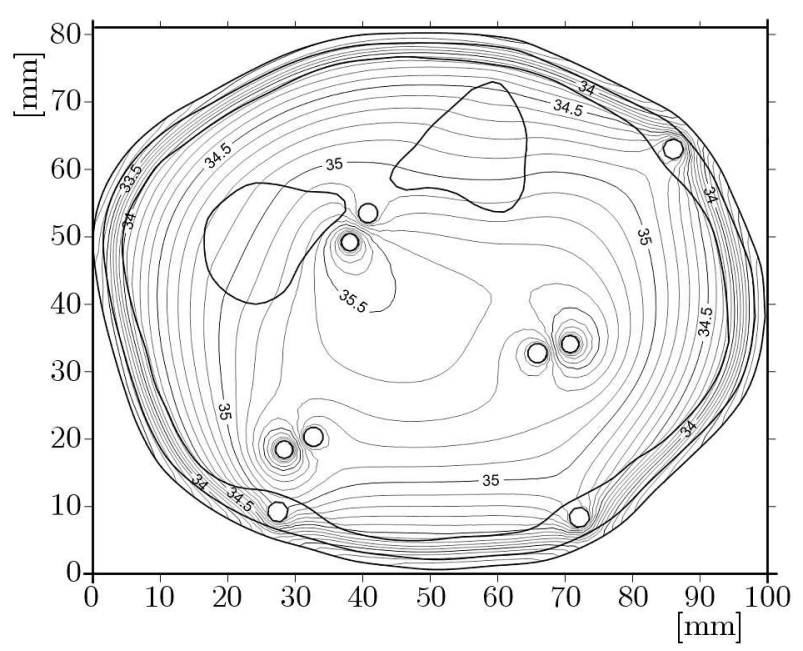

Fig. 5. The initial temperature distribution

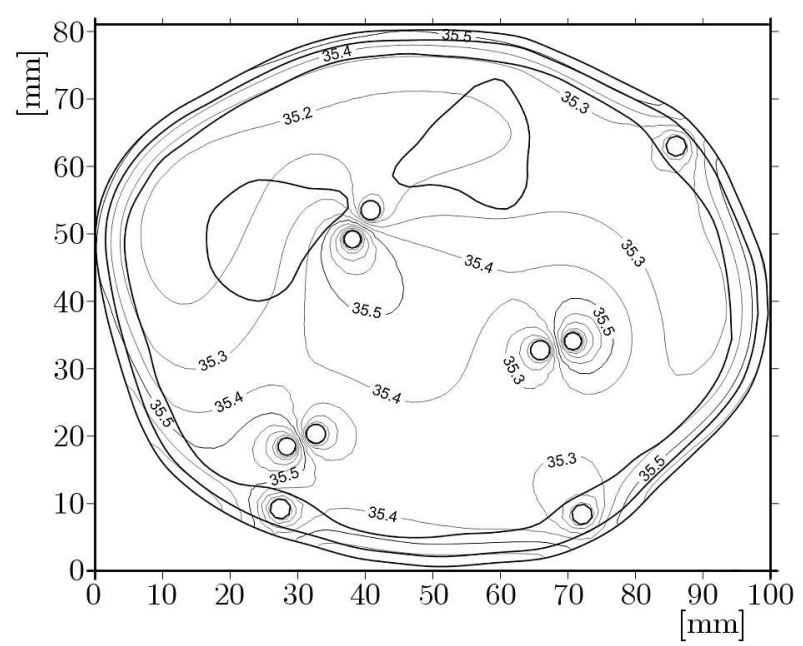

Fig. 6. Temporary solutions for $15 \mathrm{~min}$ 


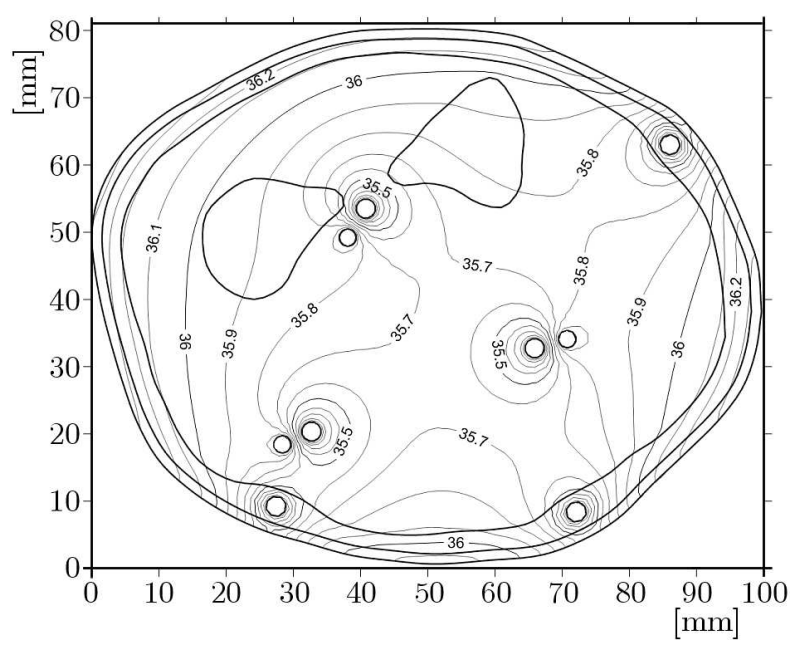

Fig. 7. Temporary solutions for $300 \mathrm{~min}$

\section{Final remarks}

In the paper, a problem from the scope of bio-heat transfer modeling is discussed. The mathematical model belongs to the group of tissue models basing on the Pennes equation. The numerical solution is obtained using the developed by the authors version of the Control Volume Method using the Voronoi tessellation. According to our opinion, the Voronoi polygons characterized by geometric properties perfectly meet the conditions required by the $2 \mathrm{D}$ control volumes. The mesh generation and the computations of the control volumes geometrical parameters are a difficult task, but the authors have developed procedures to carry out such investigations. In the case discussed, the consideration of internal heat sources has been necessary.

The numerical simulation of forearm heating in conditions of natural convection is only an example of possible application of the method proposed in the scope of bio-heat transfer. The algorithm presented can be, among others, used for numerical modeling of thermal processes proceeding in the domain of a living tissue subjected to the strong external thermal interactions (e.g. modeling of frostbites or burns). The shape and location of tissue sub-domains can be accurately reproduced (if the assumption of $2 \mathrm{D}$ approximation is acceptable). The application of the method for numerical analysis of the vascular models seems also be possible.

Acknowledgement

The paper has been a part of Project PB3/2013 sponsored by WSZOP Katowice.

\section{References}

1. Arkin H., Xu L.X, Holmes K.R., 1994, Recent development in modeling heat transfer in blood perfused tissues, IEEE Transactions on Biomedical Engineering, 41, 97-107

2. Domanski Z., Ciesielski M., Mochnacki B., 2010, Applications of control volume method using the Voronoi tessellation in numerical modeling of solidification process, [In:] Current Themes in Engineering Science 2009, Korsunsky A. (Ed.), Book Series: AIP Conference Proceedings, 1220, $17-26$

3. Fiala D., Lomas K.J., Stohrer M., 1999, A computer model of human thermoregulation for a wide range of environmental conditions: the passive system, Journal of Applied Physiology, 87, 5, 1957-1972

4. Khaled R.A., Vafai K., 2003, The role of porous media in modeling flow and heat transfer in biological tissues, International Journal of Heat and Mass Transfer, 46, 4989-5003 
5. MAJChrzak E., 2013, Application of different variants of the BEM in numerical modeling of bio-heat transfer processes, MCB: Molecular and Cellular Biomechanics, 10, 3, 201-232

6. Majchrzak E., Mochnacki B., Dziewonski M., Jasinski M., 2011, Numerical modeling of hyperthermia and hypothermia processes, Advanced Materials Research, 268-270, 257-262

7. Mochnacki B., Majchrzak E., 2003, Sensitivity of the skin tissue on the activity of external heat sources, CMES - Computer Modeling in Engineering and Sciences, 4, 3/4, 431-438

8. Mochnacki B., Piasecka-Belkhayat A., 2013, Numerical modeling of skin tissue heating using the interval finite difference method, MCB: Molecular and Cellular Biomechanics, 10, 3, 233-244

9. Nakayama A., Kuwahara F., 2008, A general bioheat transfer model based on the theory of porous media, International Journal of Heat and Mass Transfer, 51, 3190-3199

10. Okabe A., Boots B., Sugihara K., Chiu S.N., 2000, Spatial Tessellations: Concepts and Applications of Voronoi Diagrams, Second Edition, Wiley

11. Schuenke M., Schulte E., Schumacher U. Ross L., Lamperti E., 2010, General Anatomy and Musculoskeletal System (Atlas of Anatomy), Thieme, Stuttgart-New York

12. WANG H., DAI W., BEJAN A., 2007, Optimal temperature distribution in a 3D triple-layered skin structure embedded with artery and vein vasculature and induced by electromagnetic radiation, International Journal of Heat and Mass Transfer, 50, 1843-1854

13. Watson D.F., 1981, Computing the n-dimensional Delaunay tessellation with application to Voronoi polytopes, The Computer Journal, 24, 2, 167-172

14. ZHANG Y., 2009, Generalized dual-phase lag bioheat equations based on nonequilibrium heat transfer in living biological tissues, International Journal of Heat and Mass Transfer, 52, 4829-4834

15. Zhu L., Weinbaum S., 1995, A model for heat transfer from embedded blood vessels in two dimensional tissue preparations, Journal of Biomechanical Engineering, 117, 64-73

Manuscript received April 1, 2014; accepted for print April 29, 2014 